Supporting Information

\title{
Revealing Stability of Inverted Planar MA-Free Perovskite Solar Cells and Electric Field-Induced Phase Instability
}

Erjin Zheng, Zhiyin Niu, Gabriella A. Tosado, Hao Dong, Yaqoub Albrikan, and Qiuming Yu*

Department of Chemical Engineering, University of Washington, Seattle, Washington 98195, United States

*E-mail: qyu@uw.edu 


\section{Table of Contents}

\section{List of Figures}

Figure S1. The external quantum efficiency (EQE) spectrum versus wavelength and the integrated current density from the EQE spectrum of the Rb5Cs10-M device. S1

Figure S2. Normalized (a) Voc, (b) FF, and (c) J JC of the solar cells with Rb5Cs10-U and Rb5Cs10-M and Cs15-U and Cs15-M active layers stored in a $\mathrm{N}_{2}$ filled glove box in dark at room temperature with a testing interval of 3 days for 30 days. (d, e) Photocurrent density versus effective voltage of the solar cells with Cs15-U and Cs15-M active layers, respectively, by tracking the same device over 30 days S1

Figure S3. SEM image of Rb5Cs10-U perovskite thin film. EDS data were collected from the thin film background and while rod region indicated by white circles. S2

Figure S4. UPS spectra of Rb5Cs10-U and Rb5Cs10-M, and Cs15-U and Cs15-M thin films in (a) the valence band edge region and (b) the secondary electron cut-off region. Two scans were performed on each sample to ensure the consistency. S3

Figure S5. XRD patterns taken from the areas between Ag electrodes and under the electrode of the solar cells with (a-c) Cs15-M and (d-f) Cs15-U active layers. The devices have been tested in a 3-day interval for 30 days. S4

Figure S6. Survey and high-resolution Ag 3d and I 3d XPS spectra of (a-c) Rb5Cs10-U and (d-f) Rb5Cs10-M. The Ag electrodes were carefully peeled off from the devices after testing for 30 days and the XPS measurements were conducted at the Ag electrode side touching the ETL S5 
Figure S7. Normalized ToF-SIMS profiles of $\mathrm{Rb}, \mathrm{Cs}, \mathrm{FA}, \mathrm{Pb}$, and $\mathrm{Na}$ elements acquired from the areas under the electrodes and between electrodes of the solar cells tested for 30 days with

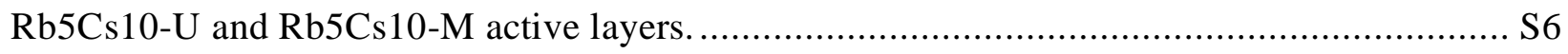

Figure S8. Normalized (a) PCE, (b) J JC, (c) Voc, and (d) FF of solar cells with a Rb5Cs10-U active layer stored in $\mathrm{N}_{2}$ filled glove box under room temperature for 30 days with the testing intervals of 3,15 , and 30 days for the first 30 days. S7

\section{List of Tables}

Table S1. Molar percentage of the elements in the Rb5Cs10-U perovskite thin film................. S2

Table S2. The work function (WF), valence band maximum (VBM), optical band gap, and conduction band minimum (CBM) of four MA-free perovskites. The VBM and optical band gaps were defined by fitting the UPS and UV-Vis spectra in a log scale. The data for each sample are the averages of two UPS scans. S3 


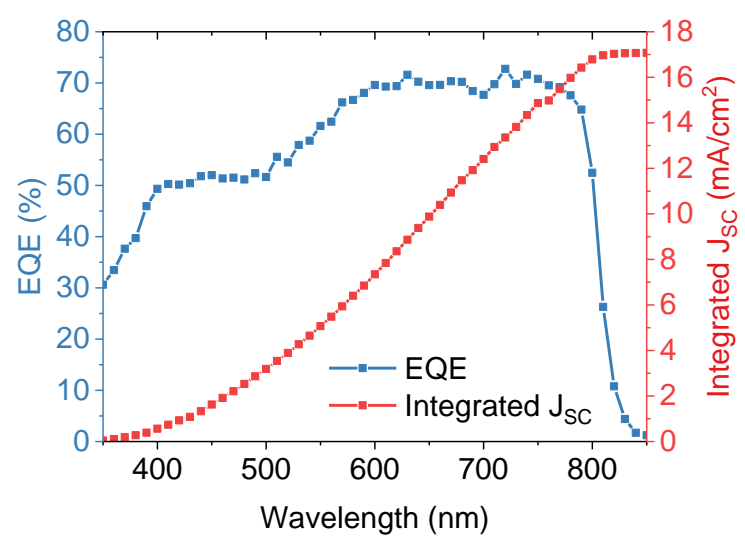

Figure S1. The external quantum efficiency (EQE) spectrum versus wavelength and the integrated current density from the EQE spectrum of the Rb5Cs10-M device.
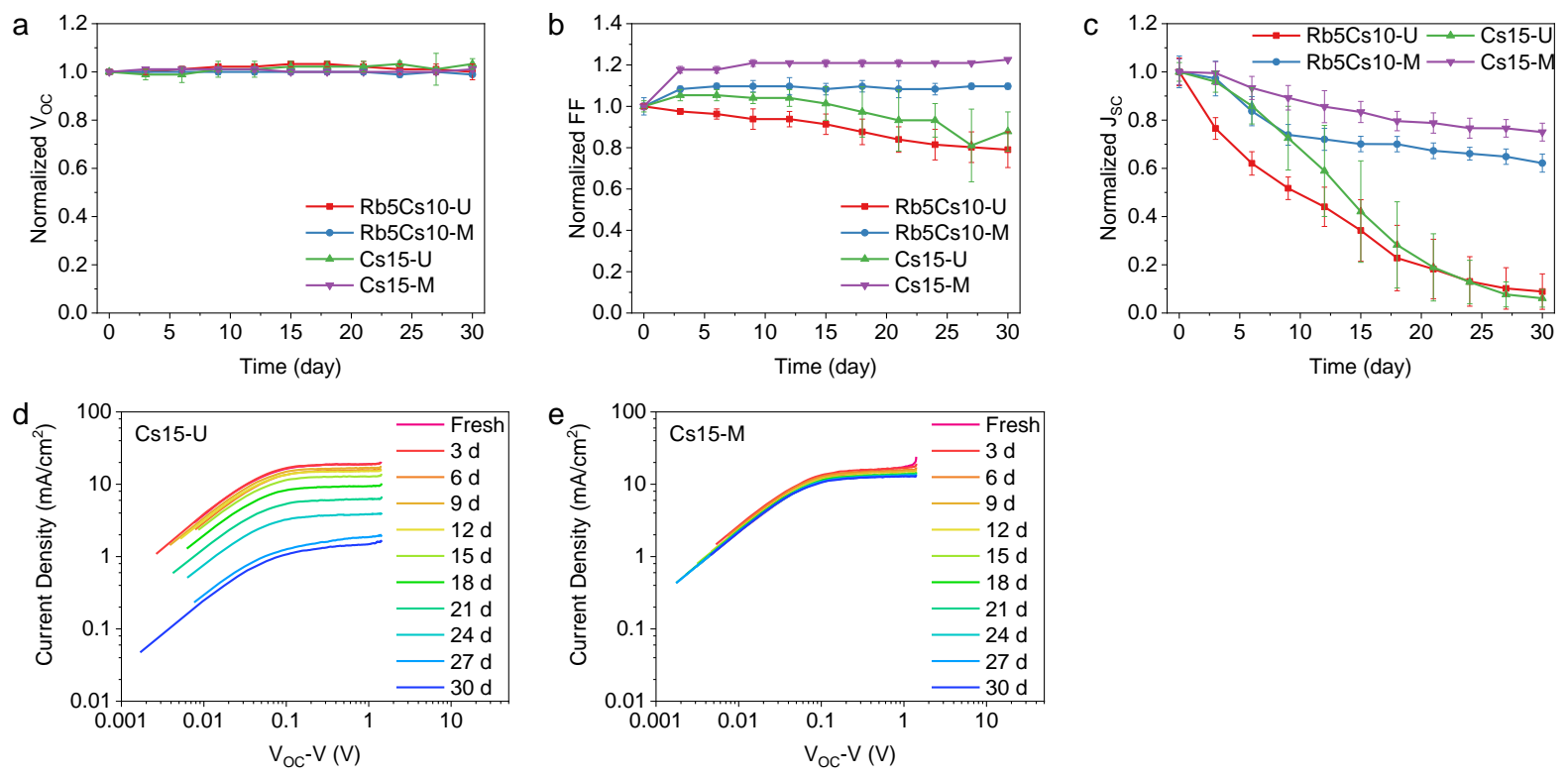

Figure S2. Normalized (a) Voc, (b) FF, and (c) JSC of the solar cells with Rb5Cs10-U and Rb5Cs10-M and Cs15-U and Cs15-M active layers stored in a $\mathrm{N}_{2}$ filled glove box in dark at room temperature with a testing interval of 3 days for 30 days. (d, e) Photocurrent density versus effective voltage of the solar cells with Cs15-U and Cs15-M active layers, respectively, by tracking the same device over 30 days. 


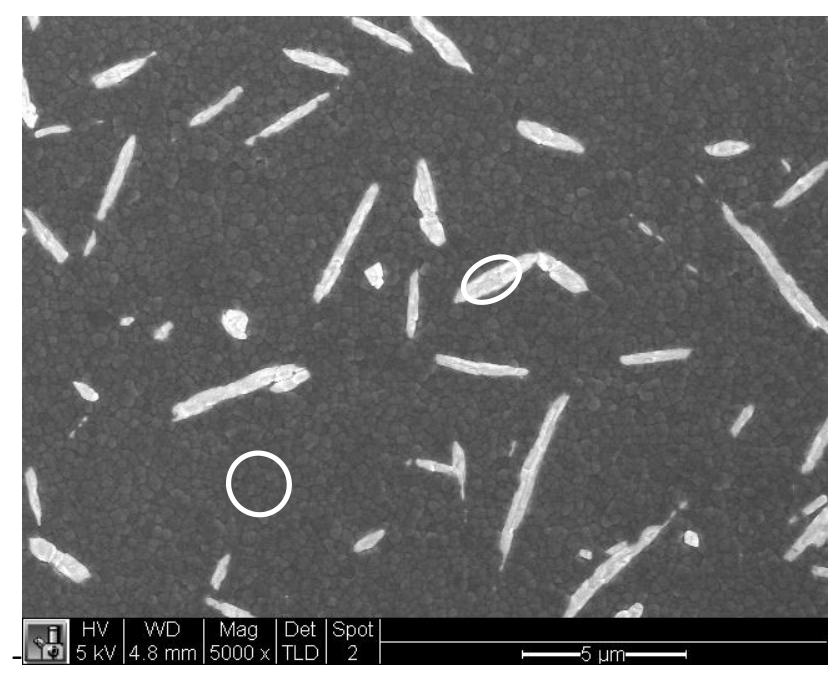

Figure S3. SEM image of Rb5Cs10-U perovskite thin film. EDS data were collected from the thin film background and while rod region indicated by white circles.

Table S1. Molar percentage of the elements in the Rb5Cs10-U perovskite thin film.

\begin{tabular}{|c|c|c|c|c|c|c|}
\hline Region & $\mathrm{I}$ & $\mathrm{Pb}$ & $\mathrm{C}$ & $\mathrm{N}$ & $\mathrm{Cs}$ & $\mathrm{Rb}$ \\
\hline Background & $30.1 \% \pm 0.3 \%$ & $11.0 \% \pm 0.2 \%$ & $37.2 \% \pm 0.4 \%$ & $20.2 \% \pm 0.7 \%$ & $1.4 \% \pm 0.1 \%$ & $0.1 \% \pm 0.1 \%$ \\
\hline White rods & $33.5 \% \pm 1.4 \%$ & $12.1 \% \pm 0.3 \%$ & $38.9 \% \pm 1.9 \%$ & $8.9 \% \pm 0.8 \%$ & $3.6 \% \pm 0.1 \%$ & $3.0 \% \pm 0.5 \%$ \\
\hline
\end{tabular}


a

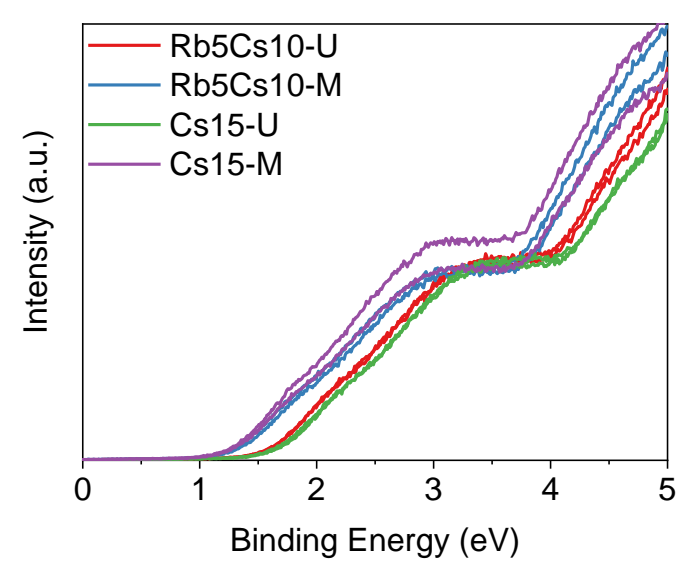

b

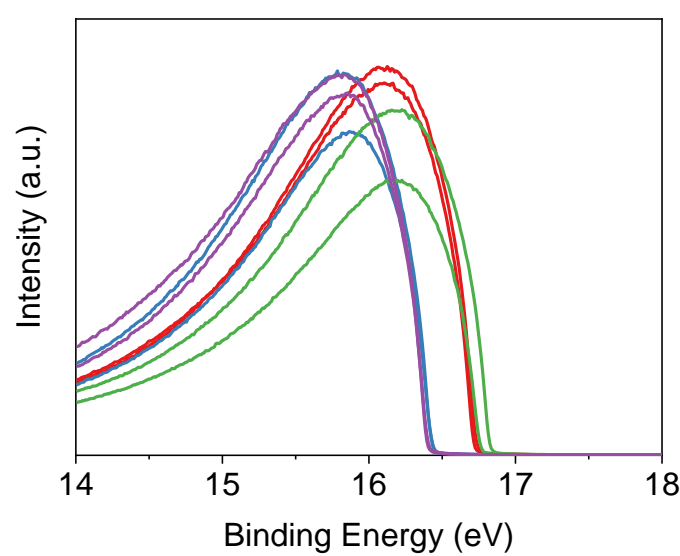

Figure S4. UPS spectra of Rb5Cs10-U and Rb5Cs10-M, and Cs15-U and Cs15-M thin films in (a) the valence band edge region and (b) the secondary electron cut-off region. Two scans were performed on each sample to ensure the consistency.

Table S2. The work function (WF), valence band maximum (VBM), optical band gap, and conduction band minimum (CBM) of four MA-free perovskites. The VBM and optical band gaps were defined by fitting the UPS and UV-Vis spectra in a log scale. The data for each sample are the averages of two UPS scans.

\begin{tabular}{|c|c|c|c|c|}
\hline & WF (eV) & VBM (eV) & Optical Bandgap (eV) & CBM (eV) \\
\hline Rb5Cs10-U & -4.41 & -5.68 & 1.49 & -4.19 \\
\hline Rb5Cs10-M & -4.69 & -5.63 & 1.49 & -4.14 \\
\hline Cs15-U & -4.34 & -5.64 & 1.51 & -4.13 \\
\hline Cs15-M & -4.72 & -5.67 & 1.51 & -4.16 \\
\hline
\end{tabular}


a

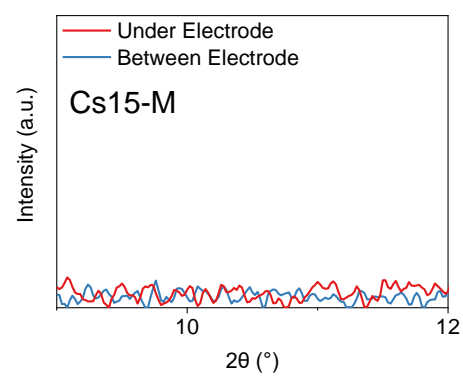

d

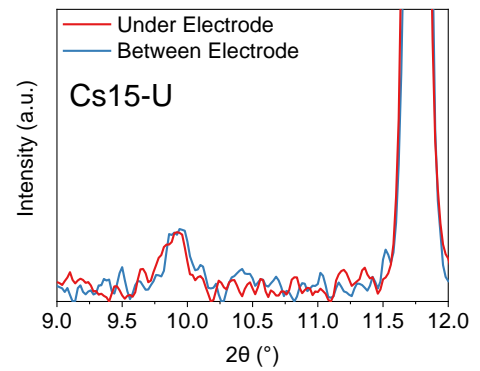

b
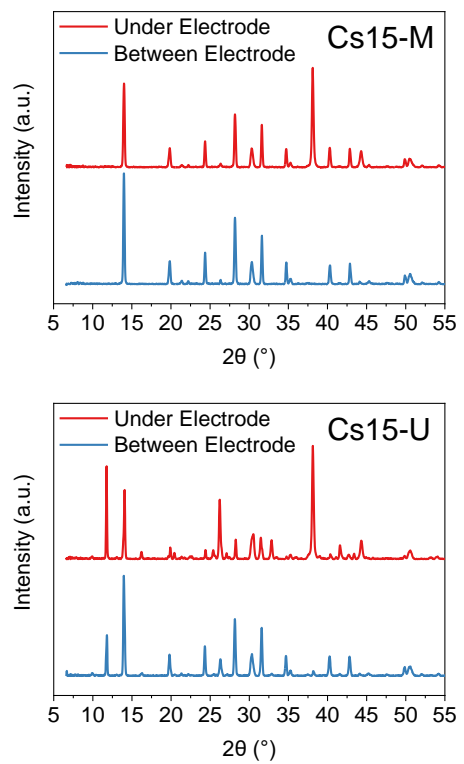
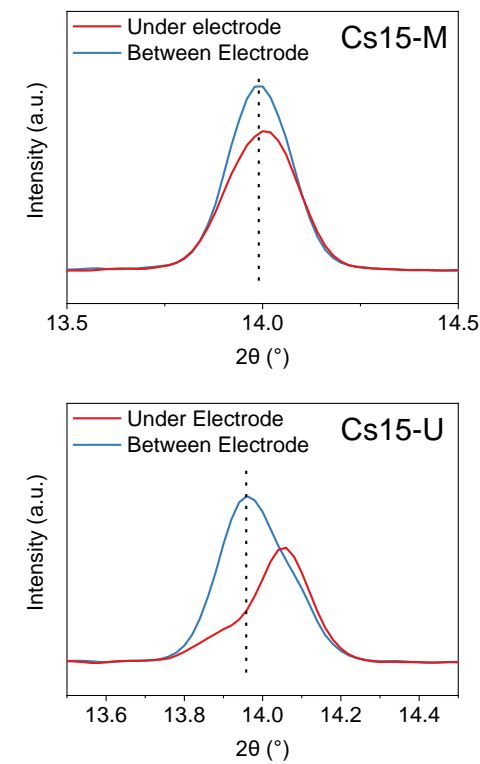

Figure S5. XRD patterns taken from the areas between Ag electrodes and under the electrode of the solar cells with (a-c) Cs15-M and (d-f) Cs15-U active layers. The devices have been tested in a 3-day interval for 30 days.

The Cs15-M and Cs15-U devices show the similar visual pictures as the Rb5Cs10-M and Rb5Cs10-U devices after testing for 30 days and their XRD patterns are shown in Figure S5. The active layer of the Cs15-M devices remains pure photoactive $\alpha$-phase in both under the electrode and between electrodes areas. Unlike Rb5Cs10-U devices, strong $\delta$-phase $\mathrm{FAPbI}_{3}$ peak at $11.76^{\circ}$, besides $\alpha$-phase $\mathrm{FAPbI}_{3}$ peaks, presents in the active layer of the $\mathrm{Cs} 15-\mathrm{U}$ device no matter under the electrode and between electrodes. The (110) peak down-shifts for the active layer between electrodes while it splits into two peaks for the active layer under the electrode, corresponding to pure $\alpha$-phase $\mathrm{FAPbI}_{3}$ and $\alpha$-phase $\mathrm{FAPbI}_{3}$ heavily doped by $\mathrm{Cs}^{+}$cation. In addition, the $\delta$-phase $\mathrm{CsPbI}_{3}$ peak at $9.94^{\circ}$ appeared in both under the electrode and between electrodes areas. Since no $\delta$-phase $\mathrm{CsPbI}_{3}$ peak was observed in the $\mathrm{Cs} 15-\mathrm{U}$ thin film after 30 days storage (Figure $2 \mathrm{~d}$ ), the 
formation of $\delta$-phase $\mathrm{CsPbI}_{3}$ after 30 days testing of the $\mathrm{C} 15-\mathrm{U}$ device could be due to the electric field induced cation migration.
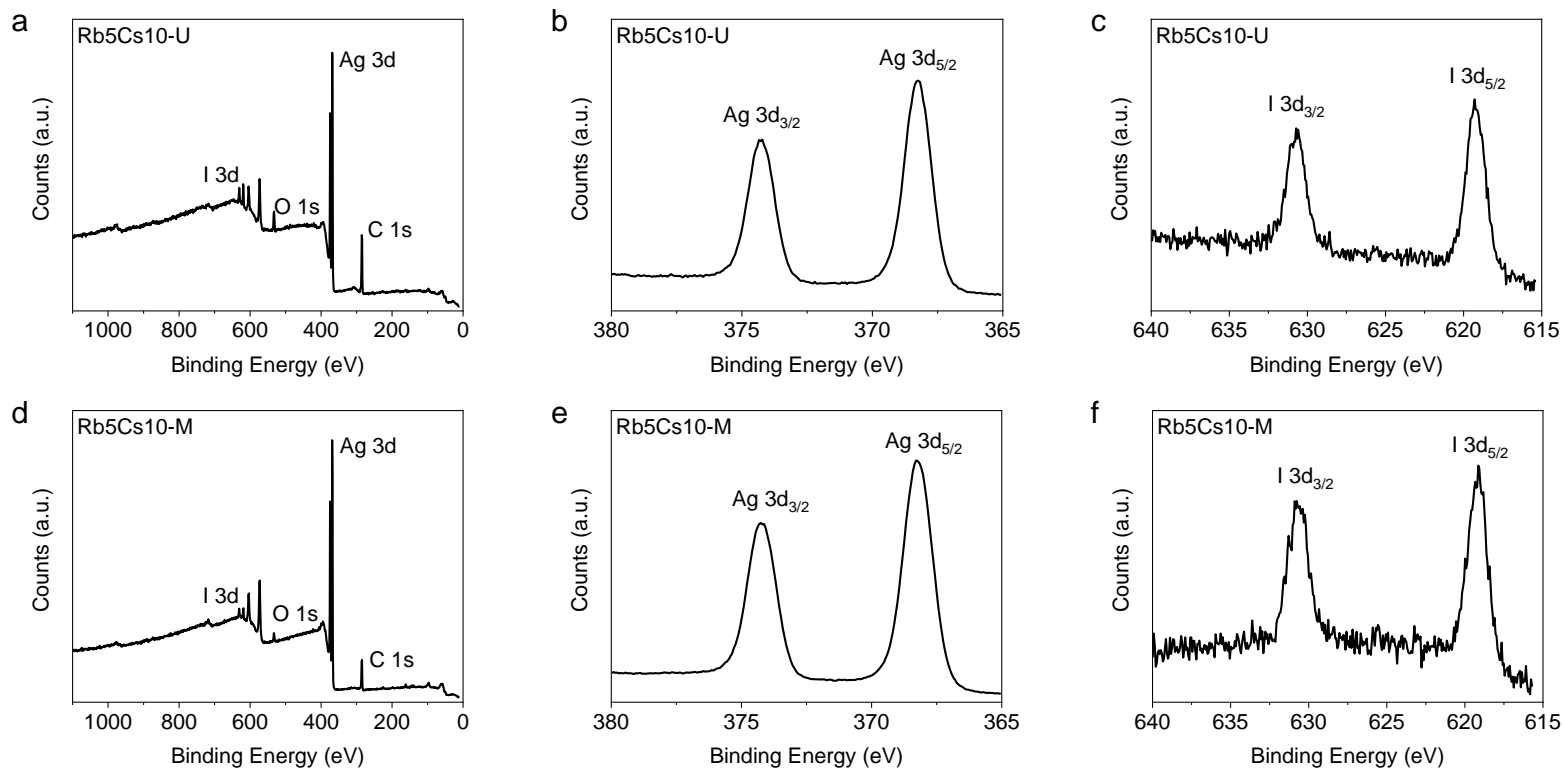

Figure S6. Survey and high-resolution Ag 3d and I 3d XPS spectra of (a-c) Rb5Cs10-U and (d-f)

Rb5Cs10-M. The Ag electrodes were carefully peeled off from the devices after testing for 30 days and the XPS measurements were conducted at the Ag electrode side touching the ETL. 

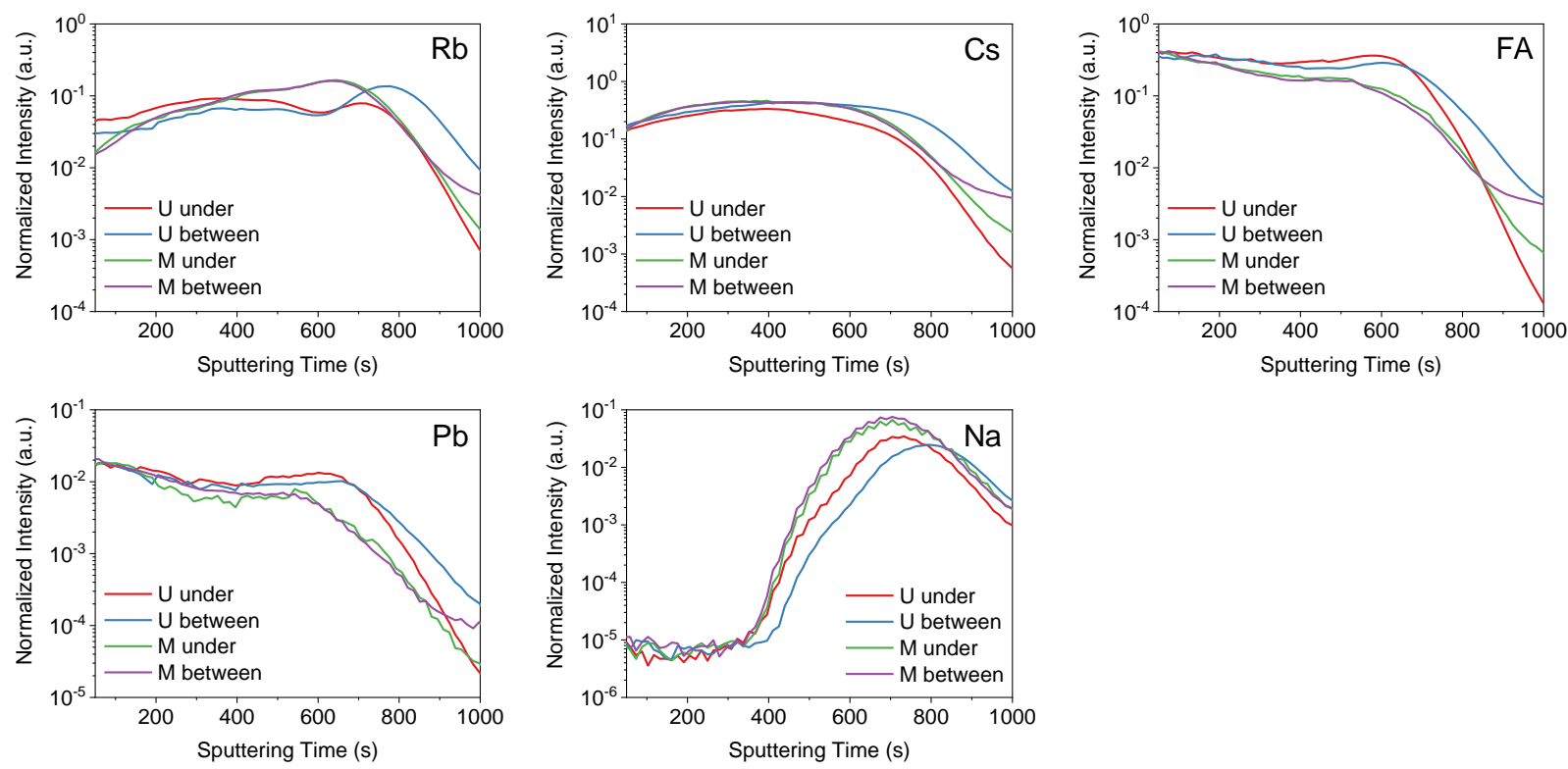

Figure S7. Normalized ToF-SIMS profiles of $\mathrm{Rb}, \mathrm{Cs}, \mathrm{FA}, \mathrm{Pb}$, and $\mathrm{Na}$ elements acquired from the areas under the electrodes and between electrodes of the solar cells tested for 30 days with Rb5Cs10-U and Rb5Cs10-M active layers. 

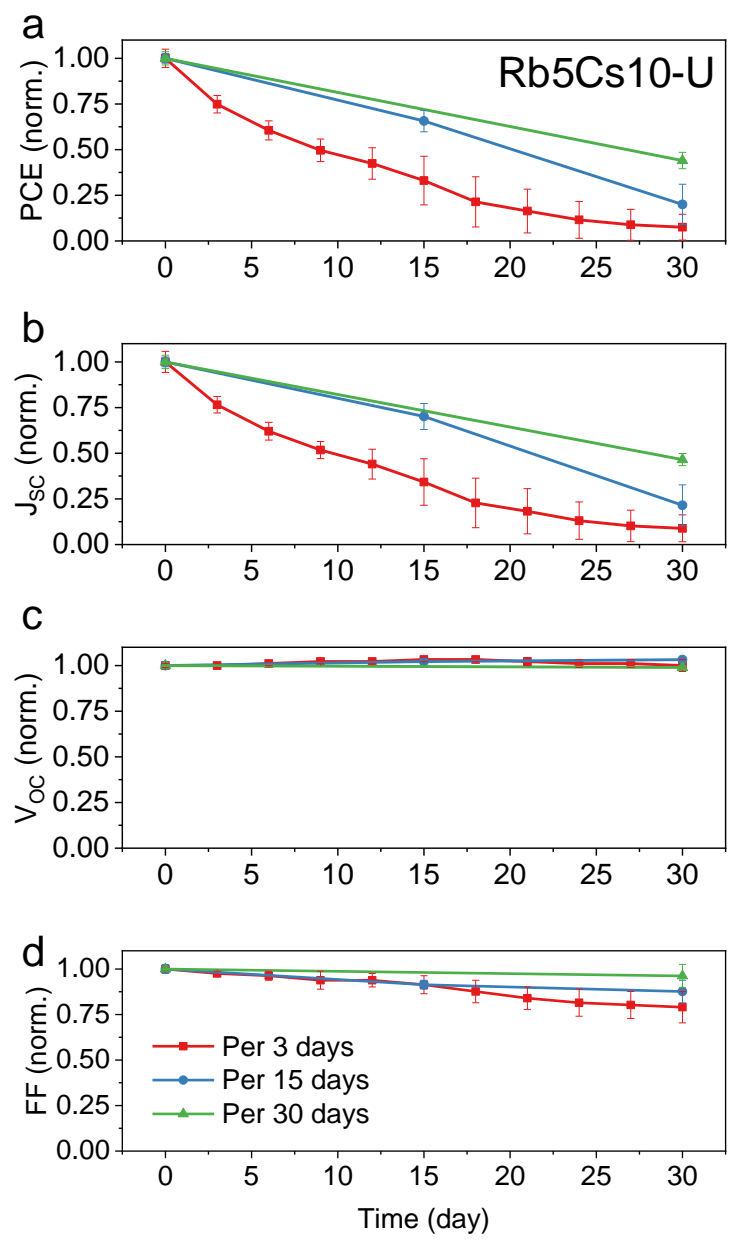

Figure S8. Normalized (a) PCE, (b) $\mathrm{J}_{\mathrm{SC}}$, (c) Voc, and (d) FF of solar cells with a Rb5Cs10-U active layer stored in $\mathrm{N}_{2}$ filled glove box under room temperature for 30 days with the testing intervals of 3,15 , and 30 days for the first 30 days. 